\title{
ADAPTIVE SAMPLING METHODS FOR NETWORK PERFORMANCE METRICS MEASUREMENT AND EVALUATION IN MPLS-BASED IP NETWORKS
}

\author{
Wenhong Ma*, James Yan+, Changcheng Huang* \\ *Optical Networks Laboratory \\ Department of Systems and Computer Engineering, Carleton University \\ 1125 Colonel By Dr., Ottawa, ON K1S 5B6, Canada \\ \{wenhongm, huang\}@sce.carleton.ca \\ +Nortel Networks \\ P.O. Box 3511, Station C \\ Ottawa, ON K1Y 4H7, Canada \\ jimyan@,nortelnetworks.com
}

\begin{abstract}
In this paper, we propose, develop and evaluate a new adaptive sampling scheme for monitoring and measuring network performance metrics in MPLS-based IP networks supporting real time applications. The paper is focused on investigating the mechanisms that can adaptively adjust the parameters of the sampling technique based on the estimated traffic rate. The performance of the proposed technique is compared with conventional sampling method by conducting simulation experiments using voice traffic patterns. Simulation results are presented to illustrate that adaptive sampling provides the potential for better monitoring, control, and management of high-performance networks with higher accuracy, lower overhead, or both.
\end{abstract}

Keywords: Adaptive sampling; Network performance metrics; Delay; IP performance measurements; MPLS

\section{INTRODUCTION}

As the Internet grows in scale and complexity, the need for network performance measurements and monitoring also increases significantly. Besides providing a good knowledge about the behavior of the network for operational purposes, measurements are critical for

CCECE 2003 - CCGEI 2003, Montreal, May/mai 2003 0-7803-7781-8/03/\$17.00 @ 2003 IEEE network planning, traffic engineering, network design optimization and SLA compliance demonstration..

Methods for measuring and monitoring network performance parameters usually fall into two categories: passive methods and active methods. Passive measurements are based on actual payload traffic in the network. They provide a statement about the treatment of the current traffic in the observed network section. However, passive measurements in high-speed networks often require the storage and processing of very large amount of data Active measurements, on the other hand, by generating the traffic needed to make the measurements, are becoming increasingly important due its great flexibility, intrinsically end-to-end in nature, and freedom from the need to involve core network switching elements. Thus active measurement methods are typically used to obtain end-to-end statistics such as latency, loss, and route availability. The method presented in this paper focuses on the application of adaptive sampling to active measurements.

In order to reduce the demand on the overhead processing of the collected data and hence to speed up related management functions, a fundamental design tradeoff in selecting a network measurement and monitoring method is to balance overhead resource costs, and the accuracy and timeliness of data. Sampling-based measurement methods, as a promising technique for reducing the amount of control data, have attracted growing interests. A new working group on this topic, PSAMP, was just formed in IETF [1], the Internet standards forum. 
Sampling techniques [2] are used to characterize the behavior of a population of elements based on a representative subset. Generally, the samples are taken periodically at some fixed interval or in some random distribution, in accordance to given characteristics. Application of three conventional sampling methods (systematic, random and stratified sampling) to network management systems and measurements are reported in [3] and [4].

Since network traffic is frequently aperiodic, the rate for any of these non-adaptive sampling techniques is typically based on the expected average network load [5], the traffic distribution, or simply to a value that will yield an acceptably small amount of overhead. If the actual traffic differs from the expected pattern, the measurements may prove inaccurate or may require excessive number of samples. For example, systematic sampling with a long sample interval is poorly suited to bursty traffic loads such as voice, which requires shorter sample intervals to accurately measure network status during high activity, and a long sampling interval providing sufficient accuracy at a minimal overhead during periods of idle activity, To address this issue, adaptive sampling techniques can be employed to dynamically adjust the sampling interval and optimize accuracy and overhead. Application of adaptive sampling to network performance measurements is presented in [6].

The main purpose of adaptive procedures is to achieve gains in precision or efficiency, compared to conventional designs of equivalent sample size, by taking advantage of observed characteristics of the population, adaptive techniques can be used to adjust the rate of sampling of network information according to the availability (or lack of) of information, thus optimizing the use of resources to acquire process and store the network nerformance data.

The remainder of the paper is organized as follows: In Section 2, the proposed adaptive sampling technique is detailed. Section 3 describes the experiments based on the application to voice over MPLS-based networks and measurement techniques used to evaluate the performance of the sampling techniques. Also, the evaluation results based on simulation are given in this section. Conclusions are provided in Section 4.

\section{ADAPTIVE SAMPLING TECHNIQUE}

The basic idea of the proposed method in this study is to use the adaptive sampling technique in conjunction with monitoring packets [7], which are inserted into the user traffic. Based on the statistics of traffic coming from different traffic sources, the adaptive sampling scheme is effective provided that the appropriate sampling interval and rate can be identified and employed. The proposed adaptive scheme has three distinct parts: a rate estimator, which is a key element in the proposed adaptive sampling for adjusting the sampling rate, sample size estimation algorithm, and sampling scheme. Details of the three main features are given below:

- A rate estimator is used to smooth out the effects of traffic burstiness as well as to be sensitive to instantaneous sending rates. A time-sliding window algorithm (TSW) [8] is employed to estimate the traffic rate. It is updated upon each packet arrival and decays over time. The average rate over the next time interval is to be determined, once the time exceeds a certain threshold, which is a pre-defined value.

- The number of monitoring packets over the fixed time interval is adaptively adjusted with the estimated traffic rate. At each fixed time interval, the sample size is updated as follows:

$$
\begin{aligned}
& \text { Sample_size }=a v g \_r a t e * \text { time_interval } / \text { (block_ } \\
& \text { size *pkt_size) }
\end{aligned}
$$

Where pkt_size is the average packet size of the user data packets and block_size is the number of user data packets between the monitoring packets. sample_size is the number of monitoring packets based on avg_rate and block_size during the appropriate chosen time interval time_interval.

- Based on the calculated sample size in the chosen fixed time interval, the stratified sampling is employed to schedule monitoring packets. This sampling method will increase the estimation accuracy by intelligent grouping, because of the higher correlation between the grouping characteristics [9].

These functions are implemented in the edge routers, which generate the monitoring packets using the proposed adaptive sampling scheme. The sending end timestamps each monitoring packet and inserts the required number of packets. The receiving end detects the monitoring packets received; timestamps them, and then process the collected data. The main performance parameters (packet delays, delay variations, packet loss ratio) can be calculated.

\section{EXPERIMENTAL EVALUATION OF SAMPLING PERFORMANCE}

\subsection{Experiment Setup}

The simulations are done with OPNET 8.0 simulation tool. As shown in Figure 1, a relatively simple but representative simulation topology based on an MPLSbased IP networks is used. MPLS is assumed because its 
path oriented nature and traffic engineering capabilities are considered as essential to providing end-to-end QoS in IP networks. Each end-host is connected to its respective edge router via a gateway; the edge routers implement the measurement functions. The edge routers are connected via a logical Label Switched Path (LSP). The LSP is the potential bottleneck as its allocated bandwidth is assumed to be less than the sum of the peak rates of the sources. Each end host group (gateway) consists of 50 voice users. Thus, the traffic traversing the simulation network is an aggregation of 50 individual flows. The goal is to measure and estimate the packet loss, delay and delay variation for the aggregate IP traffic on the LSP between edge nodes.

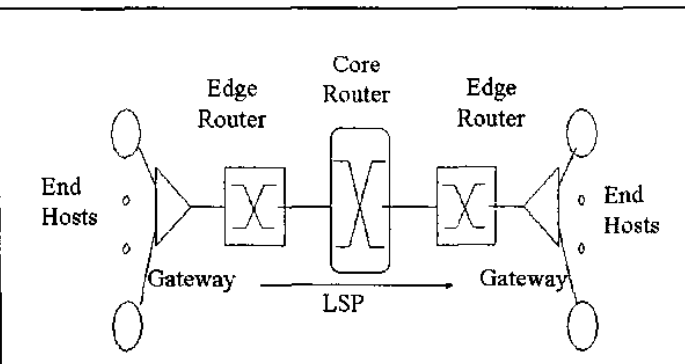

Fig.1. Simulation network topology

The traffic source model described in $[10]$ is utilized in this test. The voice source bit rate is $64 \mathrm{Kbps}$. It is assumed that the packetization period - the time to generate a packet, - is $10 \mathrm{~ms}$. Accounting for all the overhead bytes, each labeled packet has 124 bytes. Therefore, 50 voice users generate about $1.6 \mathrm{Mbps}$ traffic from the source gateway to the destination gateway. The original mean delay and mean delay variation is $49.2 \mathrm{~ms}$ and $15.2 \mathrm{~ms}$ separately. The adaptive and systematic sampling methods were then simulated and evaluated at different resolution of the measurements (The block size described in section 2 was set to $100,150,200$, and 250). The systematic sampling methods inject a monitoring packet for every fixed block size.

\subsection{Experimental Results}

The adaptive sampling technique is evaluated by comparing it to the reference sampling technique in terms of sample count and Mean Squared Error, the variance of the estimator. The block size is held constant in both methods. In this paper, sample count is defined as the amount of monitoring packets.

In general, results based on more samples have lower errors. However, as we mention earlier, the injected monitoring packets will affect the data traffic characteristics, so the less monitoring packets inserted, the lower effect on the network. As shown in Figure 2, the adaptive sampling method injects less monitoring packets than the systematic sampling for different block size.

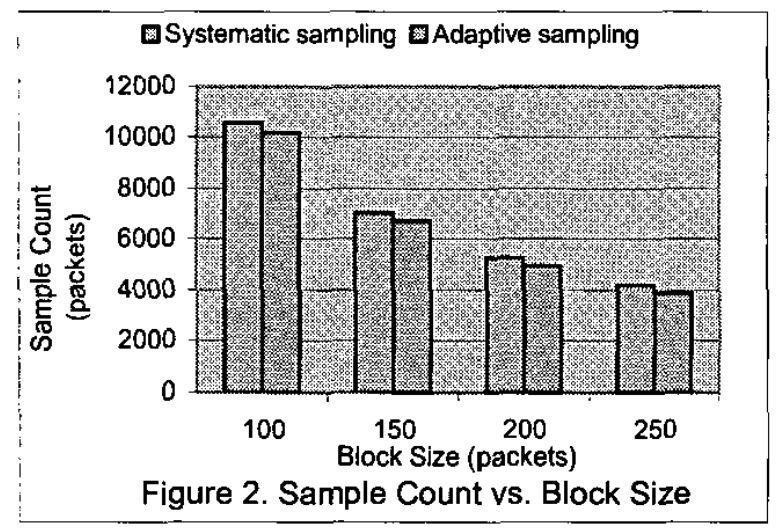

In order to compare the performance of the adaptive sampling with the systematic techniques, a useful criterion is the Mean Square Error (MSE) of the estimate, measured from the population value that is being estimated. Formally, $\operatorname{MSE}(\hat{\mu})=E(\hat{\mu}-E(\hat{\mu}))^{2}, \hat{\mu}$ is the point estimator, sample mean. $E(\hat{\mu})$ is the mean value of the estimator. Accuracy of the sampling techniques for packet delay and delay variation is assessed with the square root of the Mean Square Error, the Standard Deviation for the estimation. In Figure 3 and 4 simulation results are indicated by $S T D E V=\sqrt{M S E}$ of the mean delay and mean delay variation with the adaptive sampling, compared with the systematic sampling.

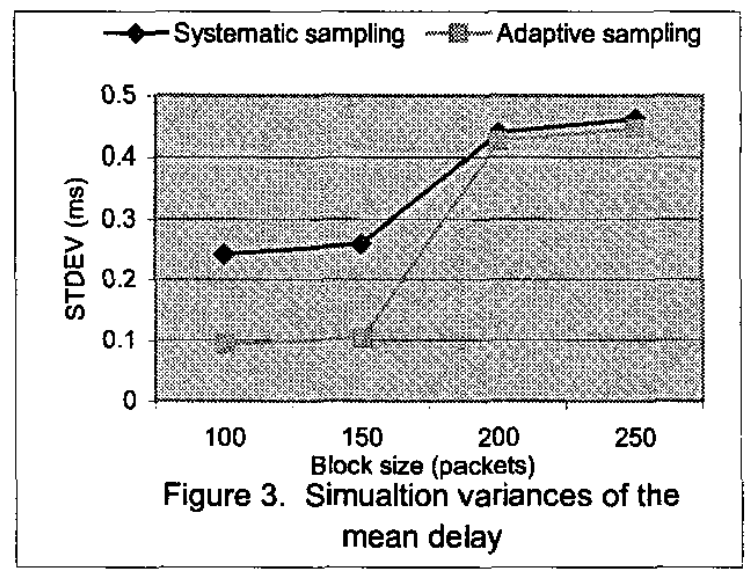

The simulation results show that measurements based on sampling are close to the actual data over different block sizes. Compared with systematic sampling using the 
same block size, adaptive sampling reduces the sample count while providing improved estimated delay and delay variation.

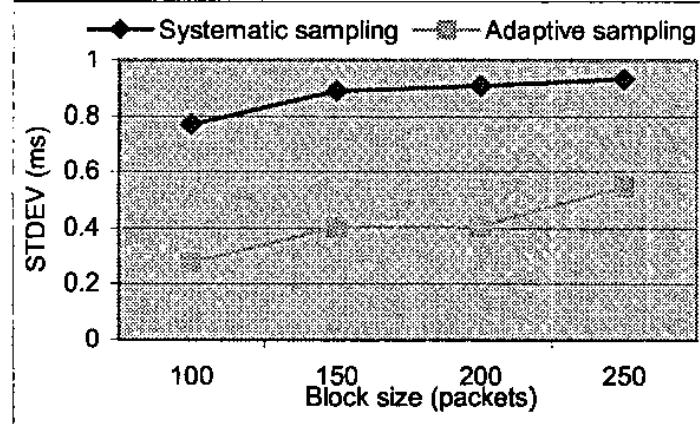

Figure 4. Simualtion variances of the mean delay variation

Figure 5 shows the packet loss ratio computed by the ratio of lost to receive monitoring packets in the two different sampling methods. Compared with the original value of $1.07 \%$ in this test, the loss ratio of the systematic samples is quite higher, while the adaptive sampling maintains almost fixed value approximate to the theory. Thus, the loss ratio can be estimated by simply counting the adaptively sent monitoring packets instead of all the packets.

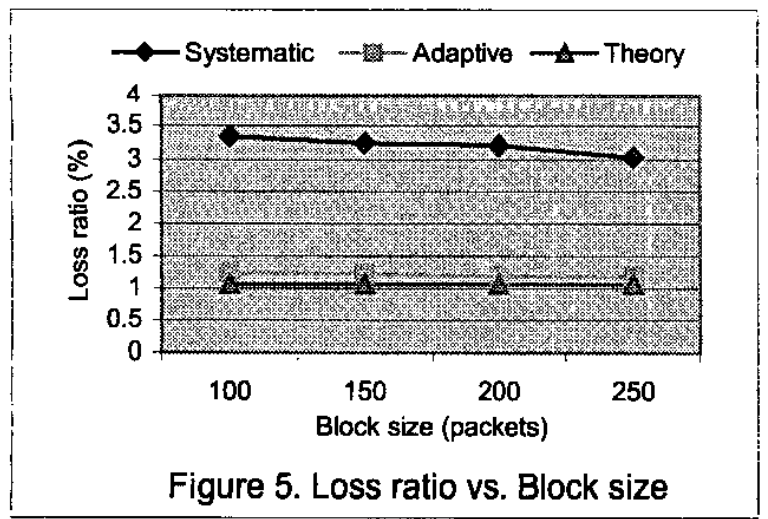

\section{CONCLUSIONS}

A new adaptive technique for network performance measurements is described in this paper. Experiments indicate that compared to systematic sampling, adaptive sampling performs well on random, bursty data such as voice traffic. This applies over a range of different block sizes. It is shown that the adaptive sampling method is able to reduce the sample count while providing better estimation accuracy. Adapting to the estimated traffic rate helps avoid large and unpredictable estimation errors found in systematic sampling.

\section{References}

[1] IETF, http://www.ietf.org

[2] W. Cochran, "Sampling Techniques", Wiley, New York, 1977.

[3] P.D. Amer, L.N. Cassel, "Management of Sampled Real-Time Network Measurements", 14th Conference on Local Computer Networks, October 1989, Minneapolis, pages 62-68, IEEE, 1989.

[4] K.C. Claffy, G.C. Polyzos, H. Braun, "Application of Sampling Methodologies to Network Traffic Characterization", Proceedings of ACM SIGCOMM'93, San Francisco, CA, USA, September 13 - 17, 1993.

[5] B. Choi, J. Park, Z.L. Zhang, "Adaptive Random Sampling for Load Change Detection", Extended Abstract in Proc. ACM SIGMETRICS June 2002.

[6] E.A. Hernandez, M.C. Chidester, and A.D. George, "Adaptive Sampling for Network Management" JNSM (Journal of Network Systems and Management): Vol. 9, No. 4, 2001

[7] T. Lindh, "An Architecture for Embedded Monitoring of QoS Parameters in IP-Based in Virtual Private Networks", Proceedings of Passive and Active Measurements (PAM) 2001, Amsterdan, 23-24 April 2001. [8] D.D. Clark, W.J. Fang, "Explicit Allocation of BestEffort Packet Delivery Service", IEEE/ACM Transactions on networking, August 1998.

[9] T. Zseby, "Deployment of Sampling Methods for SLA Validation with Non-Intrusive Measurements" Proceedings of Passive and Active Measurements (P.AM) 2002, Colorado USA, 25-26 March 2002.

[10] T. Altunbasak, "A Trade-Off Analysis for Quality of Service in Real-time Voice over IP", Master thesis the Air Force Institute of Technology, March 1999. 